\title{
Effect of Farmyard Manure and Bio-digester Liquid Manure on Soil Health under Aerobic Rice - Field Bean Cropping Sequence
}

\author{
Siddaram*, V.C. Reddy and N. Krishna Murthy \\ Department of Agronomy, University of Agricultural Sciences, Bengaluru, Karnataka, India \\ *Corresponding author
}

\author{
A B S T R A C T
}

\begin{tabular}{|l|}
\hline K e y w o r d s \\
Aerobic rice, \\
Field bean, \\
Soil health, \\
Organic carbon, \\
Microbial population, \\
Colony farming unit. \\
\hline Article Info \\
\hline $\begin{array}{l}\text { Accepted: } \\
\text { 04 April } 2017 \\
\text { Available Online: } \\
\text { 10 May } 2017\end{array}$ \\
\hline
\end{tabular}

Field experiments were conducted from 2010 to 2012 at Zonal Agricultural Research Station, Mandya to study the effect of farmyard manure and bio-digester liquid manure on the performance of aerobic rice - field bean cropping sequence. Soil was red sandy loam in texture, low in organic carbon $(0.38 \%)$ and available nitrogen $\left(215.5 \mathrm{~kg} \mathrm{ha}^{-1}\right)$, medium in available $\mathrm{P}_{2} \mathrm{O}_{5}\left(26.2 \mathrm{~kg} \mathrm{ha}^{-1}\right)$ and $\mathrm{K}_{2} \mathrm{O}\left(162.3 \mathrm{~kg} \mathrm{ha}^{-1}\right)$. Significantly higher organic carbon content $(0.51 \%)$ after harvest of aerobic rice was found with FYM $12.5 \mathrm{t}+$ BDLME to $150 \mathrm{~kg} \mathrm{~N}^{-1}$ and $0.55 \%$ with FYM $10 \mathrm{t}+$ BDLME to $35 \mathrm{~kg} \mathrm{~N} \mathrm{ha}^{-1}$ after harvest of field bean. Significantly higher available nitrogen $\left(356.2 \mathrm{~kg} \mathrm{ha}^{-1}\right), \mathrm{P}_{2} \mathrm{O}_{5}(69.5 \mathrm{~kg}$ $\left.\mathrm{ha}^{-1}\right)$ and $\mathrm{K}_{2} \mathrm{O}\left(208.0 \mathrm{~kg} \mathrm{ha}^{-1}\right)$ after harvest of aerobic rice were noticed by the application of FYM $12.5 \mathrm{t}+$ BDLME to $75 \mathrm{~kg} \mathrm{~N}^{-1}$ as compared to recommended practice. Similarly, higher available nitrogen $\left(362.3 \mathrm{~kg} \mathrm{ha}^{-1}\right), \mathrm{P}_{2} \mathrm{O}_{5}\left(87.2 \mathrm{~kg} \mathrm{ha}^{-1}\right)$ and $\mathrm{K}_{2} \mathrm{O}(227.8 \mathrm{~kg}$ $\mathrm{ha}^{-1}$ ) after harvest of field bean were noticed with of FYM $10 \mathrm{t}+$ BDLME to $20 \mathrm{~kg} \mathrm{~N}$ haas compared to recommended practice. Significantly higher population of bacteria (24.2 cfu X $10^{5} \mathrm{~g}^{-1}$ of soil), fungi (16.4 cfu X $10^{3} \mathrm{~g}^{-1} \mathrm{~s}$ of soil) and actinomycetes $\left(11.2 \mathrm{cfu} \times 10^{4}\right.$ $\mathrm{g}^{-1}$ of soil) after harvest of aerobic rice was found with FYM $12.5 \mathrm{t}+$ BDLME to $150 \mathrm{~kg} \mathrm{~N}$ $\mathrm{ha}^{-1}$ as compared to the recommended practice but was on par with FYM $12.5 \mathrm{t}+$ BDLME to $125 \mathrm{~kg} \mathrm{~N} \mathrm{ha}^{-1}$. In the same context, higher population of bacteria $\left(25.8 \mathrm{cfu} \mathrm{X} 10^{5} \mathrm{~g}^{-1}\right.$ soil), fungi (17.4 cfu X $10^{3} \mathrm{~g}^{-1}$ soil) and actinomycetes $\left(12.2 \mathrm{cfu} \times 10^{4} \mathrm{~g}^{-1}\right.$ soil) after harvest of field bean were found with the application of FYM $10 \mathrm{t}+$ BDLME to $35 \mathrm{~kg} \mathrm{~N}^{-1}$ as compared to that of recommended practice but was on par with FYM $10 \mathrm{t}+$ BDLME to 30 $\mathrm{kg} \mathrm{N} \mathrm{ha}{ }^{-1}$ but, was on par with FYM $10 \mathrm{t}+$ BDLME to $30 \mathrm{~kg} \mathrm{~N} \mathrm{ha}^{-1}$.

\section{Introduction}

Soil is the medium for life's support system. It is the foundation upon which we rely to sustain us. Out of the soil comes our food, which provides the nutrients that nourish us as individuals and as a civilization. The more we seek to improve long-term soil fertility, the more we are supporting a healthy, well-fed population in the future. Healthy, fertile soils lay the groundwork for a strong and resilient food system. Legumes are wonderful gifts of nature. Their unique ability of biological nitrogen fixation, deep root system, mobilization of insoluble soil nutrients and bringing qualitative changes in soil physical properties makes them as soil fertility restores and thereby benefits the succeeding non leguminous crop (Morey and Bagde, 1982). The practice of cereal - cereal rotation continuously might have an adverse effect on physico-chemical properties and fertility 
status of soil. Inclusion of legumes in the rotation improves the soil fertility better than cereal - cereal rotation.

Soil organisms are responsible, to a varying degree depending on the system, for performing vital functions in the soil. Soil organisms make up the diversity of life in the soil. This soil biodiversity is an important but poorly understood component of terrestrial ecosystems. Soil biodiversity is comprised of the organisms that spend all or a portion of their life cycles within the soil or on its immediate surface (including surface litter and decaying logs). Further, soil organisms represent a large fraction of global terrestrial biodiversity. They carry out a range of processes important for soil health and fertility in soils of both natural ecosystems and agricultural systems.

As farmers, we care deeply about the lands that we cultivate and want to keep them fertile for many years to come. However, for the past sixty or so years, our large-scale, "conventional" soil management techniques have largely ignored one the most fundamental requirements of the soil health i.e. life in the soil. Keeping these points in view, the field trials were carried out to study the effect of farmyard manure and biodigester liquid manure on soil health under aerobic rice - filed bean cropping sequence.

\section{Materials and Methods}

Field experiments were conducted from 2010 to 2012 at Zonal Agricultural Research Station, Mandya of the University of Agricultural Sciences, Bangalore to study the "Effect of farmyard manure and bio-digester liquid manure on the performance of aerobic rice - field bean cropping sequence". The experimental site is situated between $11^{0} 30^{\prime}$ to $13^{0} 05^{\prime}$ North latitude and $76^{\circ} 05^{\prime}$ to $77^{0}$ $45^{\prime}$ East longitude and an altitude of 695 meters above mean sea level. Soil of the experimental site was red sandy loam in texture, low in organic carbon $(0.38 \%)$ and available nitrogen $\left(215.5 \mathrm{~kg} \mathrm{ha}^{-1}\right)$, medium in available $\mathrm{P}_{2} \mathrm{O}_{5}\left(26.2 \mathrm{~kg} \mathrm{ha}^{-1}\right)$ and $\mathrm{K}_{2} \mathrm{O}(162.3$ $\mathrm{kg} \mathrm{ha}^{-1}$ ).

Representative soil samples from 0 to $30 \mathrm{~cm}$ depth were collected from each experimental plot after harvest of every crop. Soil samples thus collected were air dried in shade, powdered with wooden mallet and passed through $2 \mathrm{~mm}$ sieve and chemically analyzed for nitrogen, phosphorus, potassium content.

Available nitrogen was determined by alkaline permanganate method as outlined by Subbaiah and Asija (1959). Available phosphorus was determined by Olsen's method as outlined by Jackson (1967). Available potassium was determined by Neutral normal ammonium acetate solution using flame photometer as outlined by Jackson (1967).

Soil samples were collected from the rhizosphere of the plants at harvest. The soil samples collected were placed in a polyethylene bag and brought to laboratory and stored in refrigerator at $5^{0} \mathrm{C}$ until used for analysis. Samples were analyzed for different soil micro organism viz., total bacteria, total fungi and total actinomycetes using standard dilution plate count technique and plating on specific nutrient media.

\begin{tabular}{||l||l||}
\hline $\begin{array}{l}\text { Group of } \\
\text { microorganism }\end{array}$ & Media used \\
\hline Bacteria & Soil Extract Agar \\
\hline Fungi & $\begin{array}{l}\text { Martin's Rose Bengal Agar } \\
\text { (MRBA) }\end{array}$ \\
\hline Actinomycetes & Kuster's Agar (KA) \\
\hline \hline
\end{tabular}

\section{Results and Discussion}

Soil properties: Significantly higher organic carbon $(0.51 \%)$ content after harvest of 
aerobic rice was observed with FYM $12.5 \mathrm{t}+$ BDLME to $150 \mathrm{~kg} \mathrm{~N} \mathrm{ha}^{-1}$ closely followed by FYM $12.5 \mathrm{t}+$ BDLME to $125 \mathrm{~kg} \mathrm{~N}^{-1}(0.49$ $\%)$ and both were superior than recommended practice (FYM $10 \mathrm{t}+100: 50: 50 \mathrm{~N}: \mathrm{P}_{2} \mathrm{O}_{5}: \mathrm{K}_{2} \mathrm{O}$ $\mathrm{kg} \mathrm{ha}{ }^{-1}$ ) and other treatments (Table 1). Similarly, significantly higher organic carbon $(0.55 \%)$ content after harvest of field bean was observed with FYM $10 \mathrm{t}+$ BDLME to 35 $\mathrm{kg} \mathrm{N}$ ha $^{-1}$ closely followed by FYM $10 \mathrm{t}+$ BDLME to $30 \mathrm{~kg} \mathrm{~N} \mathrm{ha}^{-1}(0.52 \%)$ and both were superior than recommended practice $\left(\right.$ FYM $7.5 \mathrm{t}+25: 50: 25 \mathrm{~N}: \mathrm{P}_{2} \mathrm{O}_{5}: \mathrm{K}_{2} \mathrm{O} \mathrm{kg} \mathrm{ha}{ }^{-1}$ ) and other treatments (Table 2). This was mainly attributed to the contribution of carbon to soil through farmyard manure. Naveed et al., (2010) indicated that maximum value of organic matter $(1.21 \%)$ was obtained with FYM $40 \mathrm{t} \mathrm{ha}^{-1}$ followed by 1.06 per cent with FYM $20 \mathrm{t} \mathrm{ha}^{-1}$ against the minimum value $(0.93 \%)$ in recommended NPK. Reddy et al. (2011) also observed the higher soil organic carbon after harvest of rice where higher doses of FYM and BDLM were applied at Mandya, Naganahally, Bramhavar and Kathalegere. Further, Rajnish and Subhash (2011) observed that soil organic carbon was 13 per cent higher with organic nutrient management $(0.907 \%)$ than the inorganic nutrient management $(0.803 \%)$. These findings hold well in the present context.

In general, availability of nutrients in soil increased from first to second year of cultivation of both the crops. Significantly higher available nitrogen $\left(356.2 \mathrm{~kg} \mathrm{ha}^{-1}\right)$, $\mathrm{P}_{2} \mathrm{O}_{5}\left(69.5 \mathrm{~kg} \mathrm{ha}^{-1}\right)$ and $\mathrm{K}_{2} \mathrm{O}\left(208.0 \mathrm{~kg} \mathrm{ha}^{-1}\right)$ after harvest of aerobic rice were noticed by the application of FYM $12.5 \mathrm{t}+$ BDLME to $75 \mathrm{~kg} \mathrm{~N} \mathrm{ha}{ }^{-1}$ as compared to recommended practice which had lower nutrients $(262,38$ and 137) (Table 1). Similarly, higher available nitrogen (362.3 kg ha-1), phosphorus $\left(87.2 \mathrm{~kg} \mathrm{ha}^{-1}\right)$ and potassium $\left(227.8 \mathrm{~kg} \mathrm{ha}^{-1}\right)$ after harvest of filed bean were noticed with of FYM $10 \mathrm{t}+$ BDLME to $20 \mathrm{~kg} \mathrm{~N} \mathrm{ha}^{-1}$ as compared to recommended practice which had 219, 52 and $122 \mathrm{~kg} \mathrm{ha}^{-1}$, respectively (Table 2). The increase in available nitrogen content of soil could be ascribed to the increased organic matter and total nitrogen content of soil. This might also be attributed to greater multiplication of soil microbes caused by the addition of organic materials for the conversion of organically bound nitrogen to inorganic form. Nitrogen in organic form is less prone to leaching and volatilization losses. Higher $\mathrm{P}$ could be ascribed to the dissolution of native phosphorus compounds by decomposition of FYM.

Besides FYM itself could contribute considerably to this available pool upon mineralization as it contained 0.27 per cent $\mathrm{P}_{2} \mathrm{O}_{5}$. Higher $\mathrm{K}$ could be due to the direct effect of liquid manure and contribution from applied FYM to the soil pool. FYM has been reported to be a direct and ready source of potassium and also helps in minimizing the leaching loss of potassium by retaining potassium ions on exchange sites of the decomposition products. Similar results were also reported by Gopalakrishnan and Palaniappan (1992), Dikshit and Khatik (2002) and Rajshree et al., (2005).

Further, the improvement in N, P and K status of soil could be supported by the studies of Gajanana et al., (2005) who indicated that the soil health was sustainable for 25 years in FYM $\left(10 \mathrm{t} \mathrm{ha}^{-1}\right)$ applied plots or integrated plots as compared to only NPK applied plots.

Further, these treatments also recorded higher DTPA extractable $\mathrm{Zn}, \mathrm{Cu}, \mathrm{Mn}$ and $\mathrm{Fe}$ contents in the soil as compared to fertilizers only. Reddy et al., (2010) reported that at Mandya and Naganahally, soil nutrients and organic carbon status was improved by the application of FYM and bio-digester liquid manure to rice. 
Table.1 Soil properties after harvest of aerobic rice as influenced by FYM and bio-digester liquid manure

\begin{tabular}{|c|c|c|c|c|c|c|c|c|c|c|c|c|}
\hline \multirow{2}{*}{ Treatments } & \multicolumn{3}{|c|}{ Organic carbon (\%) } & \multicolumn{3}{|c|}{ Available nitrogen $\left(\mathrm{kg} \mathrm{ha}^{-1}\right)$} & \multicolumn{3}{|c|}{ Available $\mathrm{P}_{2} \mathrm{O}_{5}\left(\mathrm{~kg} \mathrm{ha}^{-1}\right)$} & \multicolumn{3}{|c|}{ Available $\mathrm{K}_{2} \mathrm{O}\left(\mathrm{kg} \mathrm{ha}^{-1}\right)$} \\
\hline & 2010 & 2011 & Pooled & 2010 & 2011 & Pooled & 2010 & 2011 & Pooled & 2010 & 2011 & Pooled \\
\hline $\mathrm{T}_{1}$ & 0.37 & 0.39 & 0.38 & 262.3 & 333.3 & 297.8 & 48.5 & 57.8 & 53.2 & 164.3 & 171.7 & 168.0 \\
\hline $\mathrm{T}_{2}$ & 0.38 & 0.40 & 0.39 & 258.3 & 331.0 & 294.7 & 48.0 & 56.7 & 52.4 & 162.0 & 170.3 & 166.2 \\
\hline $\mathrm{T}_{3}$ & 0.36 & 0.38 & 0.37 & 256.0 & 325.3 & 290.7 & 46.7 & 55.2 & 51.0 & 161.3 & 168.3 & 164.8 \\
\hline $\mathrm{T}_{4}$ & 0.38 & 0.41 & 0.40 & 280.0 & 329.7 & 304.8 & 45.2 & 54.5 & 49.9 & 158.3 & 165.0 & 161.7 \\
\hline $\mathrm{T}_{5}$ & 0.37 & 0.37 & 0.37 & 284.0 & 354.7 & 319.3 & 56.8 & 66.4 & 61.6 & 174.3 & 185.3 & 179.8 \\
\hline $\mathrm{T}_{6}$ & 0.38 & 0.40 & 0.39 & 282.3 & 350.7 & 316.5 & 54.6 & 64.1 & 59.4 & 171.0 & 181.7 & 176.3 \\
\hline $\mathrm{T}_{7}$ & 0.38 & 0.42 & 0.40 & 275.0 & 344.0 & 309.5 & 54.0 & 63.9 & 58.9 & 170.3 & 179.0 & 174.7 \\
\hline $\mathrm{T}_{8}$ & 0.39 & 0.44 & 0.41 & 280.3 & 347.0 & 313.7 & 51.2 & 61.4 & 56.3 & 167.0 & 177.7 & 172.3 \\
\hline $\mathrm{T}_{9}$ & 0.40 & 0.44 & 0.42 & 317.0 & 395.3 & 356.2 & 64.8 & 74.1 & 69.5 & 202.3 & 213.7 & 208.0 \\
\hline $\mathrm{T}_{10}$ & 0.42 & 0.46 & 0.44 & 301.0 & 379.3 & 340.2 & 61.5 & 71.5 & 66.5 & 188.0 & 198.0 & 193.0 \\
\hline $\mathrm{T}_{11}$ & 0.46 & 0.52 & 0.49 & 283.7 & 342.3 & 313.0 & 42.5 & 51.9 & 47.2 & 155.0 & 161.7 & 158.3 \\
\hline $\mathrm{T}_{12}$ & 0.48 & 0.54 & 0.51 & 281.0 & 337.7 & 309.3 & 40.8 & 50.1 & 45.5 & 153.7 & 160.3 & 157.0 \\
\hline $\mathrm{T}_{13}$ & 0.40 & 0.45 & 0.42 & 235.0 & 289.7 & 262.3 & 36.4 & 39.5 & 38.0 & 141.7 & 132.3 & 137.0 \\
\hline $\mathrm{T}_{14}$ & 0.34 & 0.31 & 0.33 & 158.7 & 64.0 & 111.0 & 16.2 & 3.3 & 9.8 & 114.0 & 48.3 & 81.2 \\
\hline Initial & & 0.38 & & & 215.5 & & & 26.2 & & & 162.3 & \\
\hline S.Em \pm & 0.02 & 0.02 & 0.02 & 9.9 & 12.2 & 11.0 & 2.1 & 1.8 & 2.0 & 8.0 & 7.0 & 7.4 \\
\hline C.D. at $5 \%$ & 0.05 & 0.07 & 0.06 & 28.7 & 35.5 & 31.1 & 6.2 & 5.4 & 5.6 & 23.3 & 20.5 & 21.0 \\
\hline
\end{tabular}

$\mathrm{T}_{1}:$ FYM $7.5 \mathrm{t}+$ BDLME to $75 \mathrm{~kg} \mathrm{~N}^{-1}$

$\mathrm{T}_{2}$ : FYM $7.5 \mathrm{t}+$ BDLME to $100 \mathrm{~kg} \mathrm{~N} \mathrm{ha}^{-1}$

$\mathrm{T}_{3}$ : FYM $7.5 \mathrm{t}+$ BDLME to $125 \mathrm{~kg} \mathrm{~N} \mathrm{ha}^{-1}$

$\mathrm{T}_{4}:$ FYM $7.5 \mathrm{t}+$ BDLME to $150 \mathrm{~kg} \mathrm{~N} \mathrm{ha}^{-1}$

$\mathrm{T}_{5}$ : FYM $10 \mathrm{t}+$ BDLME to $75 \mathrm{~kg} \mathrm{~N} \mathrm{ha}^{-1}$

FYM - Farmyard manure
$\mathrm{T}_{11}: \quad$ FYM $12.5 \mathrm{t}+$ BDLME to $125 \mathrm{~kg} \mathrm{~N} \mathrm{ha}^{-1}$ $\mathrm{T}_{12}$ : FYM $12.5 \mathrm{t}+$ BDLME to $150 \mathrm{~kg} \mathrm{~N} \mathrm{ha}^{-1}$ $\mathrm{T}_{13}:$ FYM $10 \mathrm{t}+100: 50: 50 \mathrm{~kg} \mathrm{~N}: \mathrm{P}_{2} \mathrm{O}_{5}: \mathrm{K}_{2} \mathrm{O} \mathrm{ha}^{-1}$ $\mathrm{T}_{14}$ : Absolute control

BDLME - Bio-Digester Liquid Manure Equivalent 
Table.2 Soil properties after harvest of field bean as influenced by FYM and bio-digester liquid manure

\begin{tabular}{|c|c|c|c|c|c|c|c|c|c|c|c|c|}
\hline \multirow{2}{*}{ Treatments } & \multicolumn{3}{|c|}{ Organic carbon (\%) } & \multicolumn{3}{|c|}{ Available nitrogen $\left(\mathrm{kg} \mathrm{ha}^{-1}\right)$} & \multicolumn{3}{|c|}{ Available $\mathrm{P}_{2} \mathrm{O}_{5}\left(\mathrm{~kg} \mathrm{ha}^{-1}\right)$} & \multicolumn{3}{|c|}{ Available $\mathrm{K}_{2} \mathrm{O}\left(\mathrm{kg} \mathrm{ha}^{-1}\right)$} \\
\hline & 2010 & 2011 & Pooled & 2010 & 2011 & Pooled & 2010 & 2011 & Pooled & 2010 & 2011 & Pooled \\
\hline $\mathrm{T}_{1}$ & 0.38 & 0.40 & 0.39 & 268.7 & 345.0 & 306.8 & 55.1 & 86.1 & 70.6 & 181.3 & 193.0 & 187.2 \\
\hline $\mathrm{T}_{2}$ & 0.39 & 0.41 & 0.40 & 265.0 & 340.0 & 302.5 & 53.8 & 85.5 & 69.7 & 178.7 & 188.7 & 183.7 \\
\hline $\mathrm{T}_{3}$ & 0.36 & 0.39 & 0.38 & 263.7 & 335.3 & 299.5 & 52.3 & 83.9 & 68.1 & 176.0 & 186.3 & 181.2 \\
\hline $\mathrm{T}_{4}$ & 0.39 & 0.42 & 0.40 & 255.3 & 330.7 & 293.0 & 51.8 & 83.3 & 67.6 & 172.7 & 181.7 & 177.2 \\
\hline $\mathrm{T}_{5}$ & 0.37 & 0.38 & 0.38 & 292.7 & 371.7 & 332.2 & 63.3 & 94.3 & 78.8 & 193.7 & 209.0 & 201.3 \\
\hline $\mathrm{T}_{6}$ & 0.39 & 0.42 & 0.40 & 287.0 & 370.3 & 328.7 & 60.8 & 92.2 & 76.5 & 191.0 & 204.7 & 197.8 \\
\hline $\mathrm{T}_{7}$ & 0.40 & 0.44 & 0.42 & 282.0 & 363.0 & 322.5 & 59.7 & 90.7 & 75.2 & 188.3 & 202.0 & 195.2 \\
\hline $\mathrm{T}_{8}$ & 0.40 & 0.47 & 0.44 & 274.3 & 359.0 & 316.7 & 57.8 & 89.1 & 73.5 & 185.7 & 197.3 & 191.5 \\
\hline $\mathrm{T}_{9}$ & 0.42 & 0.48 & 0.45 & 321.0 & 403.7 & 362.3 & 70.2 & 104.3 & 87.2 & 220.3 & 235.3 & 227.8 \\
\hline $\mathrm{T}_{10}$ & 0.43 & 0.50 & 0.46 & 308.0 & 397.0 & 352.5 & 68.1 & 100.4 & 84.2 & 206.0 & 221.0 & 213.5 \\
\hline $\mathrm{T}_{11}$ & 0.47 & 0.56 & 0.52 & 250.7 & 328.0 & 289.3 & 49.3 & 81.6 & 65.5 & 170.0 & 178.7 & 174.3 \\
\hline $\mathrm{T}_{12}$ & 0.51 & 0.59 & 0.55 & 244.3 & 319.3 & 281.8 & 47.4 & 79.1 & 63.3 & 167.3 & 175.3 & 171.3 \\
\hline $\mathrm{T}_{13}$ & 0.43 & 0.47 & 0.45 & 198.0 & 240.3 & 219.2 & 38.4 & 66.5 & 52.4 & 125.7 & 119.0 & 122.3 \\
\hline $\mathrm{T}_{14}$ & 0.33 & 0.27 & 0.30 & 110.7 & 24.3 & 67.5 & 10.8 & 1.9 & 6.4 & 82.2 & 14.2 & 48.2 \\
\hline S.Em \pm & 0.02 & 0.02 & 0.02 & 7.2 & 9.0 & 8.4 & 2.1 & 2.3 & 2.2 & 8.3 & 7.6 & 8.5 \\
\hline C.D. at $5 \%$ & 0.06 & 0.07 & 0.07 & 21.0 & 26.1 & 23.7 & 6.0 & 6.6 & 6.1 & 24.2 & 22.1 & 24.1 \\
\hline
\end{tabular}

$\mathrm{T}_{1}:$ FYM $5 \mathrm{t}+$ BDLME to $20 \mathrm{~kg} \mathrm{~N} \mathrm{ha}^{-1}$

$\mathrm{T}_{2}$ : FYM $5 \mathrm{t}+$ BDLME to $25 \mathrm{~kg} \mathrm{~N} \mathrm{ha}^{-1}$

$\mathrm{T}_{3}:$ FYM $5 \mathrm{t}+$ BDLME to $30 \mathrm{~kg} \mathrm{~N}^{-1}$

$\mathrm{T}_{4}$ : FYM $5 \mathrm{t}+$ BDLME to $35 \mathrm{~kg} \mathrm{~N} \mathrm{ha}^{-1}$

$\mathrm{T}_{5}$ : FYM $7.5 \mathrm{t}+$ BDLME to $20 \mathrm{~kg} \mathrm{~N} \mathrm{ha}^{-1}$

FYM - Farmyard manure
$\mathrm{T}_{6}$ : FYM $7.5 \mathrm{t}+$ BDLME to $25 \mathrm{~kg} \mathrm{~N} \mathrm{ha}^{-1}$ $\mathrm{T}_{7}$ : FYM $7.5 \mathrm{t}+$ BDLME to $30 \mathrm{~kg} \mathrm{~N} \mathrm{ha}^{-1}$ $\mathrm{T}_{8}:$ FYM $7.5 \mathrm{t}+$ BDLME to $35 \mathrm{~kg} \mathrm{~N} \mathrm{ha}^{-1}$ $\mathrm{T}_{9}$ : FYM $10 \mathrm{t}+$ BDLME to $20 \mathrm{~kg} \mathrm{~N}^{-1}$ $\mathrm{T}_{10}$ : FYM $10 \mathrm{t}+$ BDLME to $25 \mathrm{~kg} \mathrm{~N} \mathrm{ha}^{-1}$ CFU - Colony Forming Unit
$\mathrm{T}_{11}$ : FYM $10 \mathrm{t}+$ BDLME to $30 \mathrm{~kg} \mathrm{~N}^{-1}$ $\mathrm{T}_{12}$ : FYM $10 \mathrm{t}+$ BDLME to $35 \mathrm{~kg} \mathrm{~N} \mathrm{ha}^{-1}$ $\mathrm{T}_{13}:$ FYM $7.5 \mathrm{t}+25: 50: 25 \mathrm{~kg} \mathrm{~N}: \mathrm{P}_{2} \mathrm{O}_{5}: \mathrm{K}_{2} \mathrm{O}$ ha $^{-1}$ $\mathrm{T}_{14}$ : Absolute control BDLME - Bio-Digester Liquid Manure Equivalent 
Table.3 Population of soil microorganisms after harvest of aerobic rice as influenced by FYM and bio-digester liquid manure

\begin{tabular}{|c|c|c|c|c|c|c|c|c|c|}
\hline \multirow{2}{*}{ Treatments } & \multicolumn{3}{|c|}{ Bacteria (cfu $\times 10^{5} \mathrm{~g}^{-1}$ of soil) } & \multicolumn{3}{|c|}{ Fungi (cfu x $10^{3} \mathrm{~g}^{-1}$ of soil) } & \multicolumn{3}{|c|}{ Actinomycetes (cfu $\times 10^{4} \mathrm{~g}^{-1}$ of soil) } \\
\hline & 2010 & 2011 & Pooled & 2010 & 2011 & Pooled & 2010 & 2011 & Pooled \\
\hline $\mathrm{T}_{1}$ & 12.1 & 14.3 & 13.2 & 9.5 & 11.0 & 10.3 & 6.5 & 7.4 & 7.0 \\
\hline $\mathrm{T}_{2}$ & 13.0 & 15.5 & 14.3 & 10.1 & 11.6 & 10.9 & 6.7 & 7.8 & 7.3 \\
\hline $\mathrm{T}_{3}$ & 13.3 & 15.9 & 14.6 & 11.2 & 12.5 & 11.9 & 7.2 & 7.9 & 7.6 \\
\hline $\mathrm{T}_{4}$ & 13.6 & 16.5 & 15.1 & 11.7 & 13.1 & 12.4 & 7.3 & 8.3 & 7.8 \\
\hline $\mathrm{T}_{5}$ & 14.1 & 17.0 & 15.6 & 11.9 & 13.3 & 12.6 & 7.4 & 8.7 & 8.1 \\
\hline $\mathrm{T}_{6}$ & 14.3 & 17.4 & 15.8 & 12.3 & 13.9 & 13.1 & 7.7 & 8.9 & 8.3 \\
\hline $\mathrm{T}_{7}$ & 15.8 & 18.6 & 17.2 & 12.5 & 14.5 & 13.5 & 8.1 & 9.3 & 8.7 \\
\hline $\mathrm{T}_{8}$ & 16.3 & 20.7 & 18.5 & 12.7 & 14.5 & 13.6 & 8.4 & 9.7 & 9.1 \\
\hline $\mathrm{T}_{9}$ & 18.5 & 21.4 & 20.0 & 13.1 & 14.8 & 14.0 & 8.5 & 10.0 & 9.3 \\
\hline $\mathrm{T}_{10}$ & 19.4 & 22.8 & 21.1 & 13.2 & 15.1 & 14.2 & 8.8 & 10.3 & 9.5 \\
\hline $\mathrm{T}_{11}$ & 21.3 & 24.9 & 23.1 & 14.8 & 16.7 & 15.8 & 9.9 & 11.5 & 10.7 \\
\hline $\mathrm{T}_{12}$ & 22.5 & 25.8 & 24.2 & 15.2 & 17.5 & 16.4 & 10.2 & 12.1 & 11.2 \\
\hline $\mathrm{T}_{13}$ & 11.7 & 12.2 & 11.9 & 8.9 & 9.5 & 9.2 & 6.2 & 6.7 & 6.4 \\
\hline $\mathrm{T}_{14}$ & 9.2 & 7.2 & 8.2 & 4.9 & 4.0 & 4.5 & 3.9 & 3.3 & 3.6 \\
\hline Initial & \multicolumn{3}{|c|}{11.2} & \multicolumn{3}{|c|}{8.6} & \multicolumn{3}{|c|}{6.1} \\
\hline S.Em \pm & 0.8 & 0.7 & 0.8 & 0.5 & 0.6 & 0.6 & 0.4 & 0.4 & 0.5 \\
\hline C.D. at $5 \%$ & 2.2 & 2.2 & 2.2 & 1.4 & 1.7 & 1.6 & 1.0 & 1.2 & 1.4 \\
\hline
\end{tabular}

$\mathrm{T}_{1}:$ FYM $7.5 \mathrm{t}+$ BDLME to $75 \mathrm{~kg} \mathrm{~N} \mathrm{ha}^{-1}$

$\mathrm{T}_{2}$ : FYM $7.5 \mathrm{t}+$ BDLME to $100 \mathrm{~kg} \mathrm{~N} \mathrm{ha}^{-1}$

$\mathrm{T}_{3}:$ FYM $7.5 \mathrm{t}+$ BDLME to $125 \mathrm{~kg} \mathrm{~N} \mathrm{ha}^{-1}$

$\mathrm{T}_{4}:$ FYM $7.5 \mathrm{t}+$ BDLME to $150 \mathrm{~kg} \mathrm{~N} \mathrm{ha}^{-1}$

$\mathrm{T}_{5}$ : FYM $10 \mathrm{t}+$ BDLME to $75 \mathrm{~kg} \mathrm{~N} \mathrm{ha}^{-1}$

FYM - Farmyard manure
$\mathrm{T}_{6}:$ FYM $10 \mathrm{t}+$ BDLME to $100 \mathrm{~kg} \mathrm{~N} \mathrm{ha}^{-1}$

$\mathrm{T}_{7}$ : FYM $10 \mathrm{t}+$ BDLME to $125 \mathrm{~kg} \mathrm{~N} \mathrm{ha}^{-1}$

$\mathrm{T}_{8}:$ FYM $10 \mathrm{t}+$ BDLME to $150 \mathrm{~kg} \mathrm{~N}^{-1}$

$\mathrm{T}_{9}$ : FYM $12.5 \mathrm{t}+$ BDLME to $75 \mathrm{~kg} \mathrm{~N} \mathrm{ha}^{-1}$

$\mathrm{T}_{10}$ : FYM $12.5 \mathrm{t}+$ BDLME to $100 \mathrm{~kg} \mathrm{~N} \mathrm{ha}^{-1}$

CFU - Colony Forming Unit
$\mathrm{T}_{11}$ : FYM $12.5 \mathrm{t}+$ BDLME to $125 \mathrm{~kg} \mathrm{~N} \mathrm{ha}^{-1}$

$\mathrm{T}_{12}$ : FYM $12.5 \mathrm{t}+$ BDLME to $150 \mathrm{~kg} \mathrm{~N} \mathrm{ha}^{-1}$

$\mathrm{T}_{13}: \quad F Y M 10 \mathrm{t}+100: 50: 50 \mathrm{~kg} \mathrm{~N}: \mathrm{P}_{2} \mathrm{O}_{5}: \mathrm{K}_{2} \mathrm{O} \mathrm{ha}^{-1}$

$\mathrm{T}_{14}$ : Absolute control

BDLME - Bio-Digester Liquid Manure

Equivalent 
Table.4 Population of soil microorganisms after harvest of field bean as influenced by FYM and bio-digester liquid manure

\begin{tabular}{|c|c|c|c|c|c|c|c|c|c|}
\hline \multirow{2}{*}{ Treatments } & \multicolumn{3}{|c|}{ Bacteria (cfu x $10^{5} \mathrm{~g}^{-1}$ of soil) } & \multicolumn{3}{|c|}{ Fungi (cfu $\times 10^{3} \mathrm{~g}^{-1}$ of soil) } & \multicolumn{3}{|c|}{ Actinomycetes (cfu $\times 10^{4} \mathrm{~g}^{-1}$ of soil } \\
\hline & 2010 & 2011 & Pooled & 2010 & 2011 & Pooled & 2010 & 2011 & Pooled \\
\hline $\mathrm{T}_{1}$ & 13.6 & 15.7 & 14.7 & 10.6 & 12.1 & 11.4 & 7.1 & 8.1 & 7.6 \\
\hline $\mathrm{T}_{2}$ & 14.4 & 16.5 & 15.5 & 11.0 & 12.8 & 11.9 & 7.2 & 8.5 & 7.9 \\
\hline $\mathrm{T}_{3}$ & 14.7 & 17.0 & 15.9 & 12.1 & 13.5 & 12.8 & 7.6 & 8.6 & 8.1 \\
\hline $\mathrm{T}_{4}$ & 15.3 & 17.8 & 16.5 & 12.5 & 14.3 & 13.4 & 7.7 & 9.1 & 8.4 \\
\hline $\mathrm{T}_{5}$ & 15.8 & 18.6 & 17.2 & 12.8 & 14.4 & 13.6 & 7.8 & 9.5 & 8.7 \\
\hline $\mathrm{T}_{6}$ & 15.8 & 19.0 & 17.4 & 13.2 & 14.9 & 14.1 & 8.4 & 9.8 & 9.1 \\
\hline $\mathrm{T}_{7}$ & 17.3 & 20.1 & 18.7 & 13.7 & 15.3 & 14.5 & 8.8 & 10.4 & 9.6 \\
\hline $\mathrm{T}_{8}$ & 19.5 & 22.1 & 20.8 & 13.8 & 15.6 & 14.7 & 9.2 & 10.5 & 9.8 \\
\hline $\mathrm{T}_{9}$ & 20.1 & 22.8 & 21.4 & 14.2 & 15.9 & 15.0 & 9.3 & 11.0 & 10.2 \\
\hline $\mathrm{T}_{10}$ & 21.4 & 24.1 & 22.8 & 14.8 & 16.2 & 15.5 & 9.5 & 11.1 & 10.3 \\
\hline $\mathrm{T}_{11}$ & 23.8 & 26.0 & 24.9 & 15.9 & 17.7 & 16.8 & 10.8 & 12.4 & 11.6 \\
\hline $\mathrm{T}_{12}$ & 24.5 & 27.2 & 25.8 & 16.6 & 18.2 & 17.4 & 11.1 & 13.3 & 12.2 \\
\hline $\mathrm{T}_{13}$ & 13.0 & 11.5 & 12.3 & 10.2 & 9.1 & 9.7 & 7.0 & 6.4 & 6.7 \\
\hline $\mathrm{T}_{14}$ & 8.5 & 6.5 & 7.5 & 4.4 & 3.7 & 4.1 & 3.6 & 3.0 & 3.3 \\
\hline S.Em \pm & 0.82 & 0.82 & 0.83 & 0.44 & 0.64 & 0.64 & 0.30 & 0.64 & 0.50 \\
\hline C.D. at $5 \%$ & 2.40 & 2.38 & 2.36 & 1.28 & 1.85 & 1.82 & 0.86 & 1.86 & 1.42 \\
\hline
\end{tabular}

$\mathrm{T}_{1}:$ FYM $5 \mathrm{t}+$ BDLME to $20 \mathrm{~kg} \mathrm{~N} \mathrm{ha}^{-1}$

$\mathrm{T}_{2}:$ FYM $5 \mathrm{t}+\mathrm{BDLME}$ to $25 \mathrm{~kg} \mathrm{~N} \mathrm{ha}^{-1}$

$\mathrm{T}_{3}:$ FYM $5 \mathrm{t}+$ BDLME to $30 \mathrm{~kg} \mathrm{~N}^{-1}$

$\mathrm{T}_{4}:$ FYM $5 \mathrm{t}+$ BDLME to $35 \mathrm{~kg} \mathrm{~N}^{-1}$

$\mathrm{T}_{5}$ : FYM $7.5 \mathrm{t}+$ BDLME to $20 \mathrm{~kg} \mathrm{~N}^{-1}$

FYM - Farmyard manure
$\mathrm{T}_{6}:$ FYM $7.5 \mathrm{t}+$ BDLME to $25 \mathrm{~kg} \mathrm{~N} \mathrm{ha}^{-1}$

$\mathrm{T}_{7}:$ FYM $7.5 \mathrm{t}+$ BDLME to $30 \mathrm{~kg} \mathrm{~N}^{-1}$

$\mathrm{T}_{8}:$ FYM $7.5 \mathrm{t}+$ BDLME to $35 \mathrm{~kg} \mathrm{~N} \mathrm{ha}^{-1}$

$\mathrm{T}_{9}$ : FYM $10 \mathrm{t}+$ BDLME to $20 \mathrm{~kg} \mathrm{~N}^{-1}$

$\mathrm{T}_{10}$ : FYM $10 \mathrm{t}+$ BDLME to $25 \mathrm{~kg} \mathrm{~N} \mathrm{ha}^{-1}$

$\mathrm{CFU}$ - Colony Forming Unit
$\mathrm{T}_{11}:$ FYM $10 \mathrm{t}+$ BDLME to $30 \mathrm{~kg} \mathrm{~N} \mathrm{ha}^{-1}$

$\mathrm{T}_{12}$ : FYM $10 \mathrm{t}+$ BDLME to $35 \mathrm{~kg} \mathrm{~N}^{-1}$

$\mathrm{T}_{13}:$ FYM $7.5 \mathrm{t}+25: 50: 25 \mathrm{~kg} \mathrm{~N}: \mathrm{P}_{2} \mathrm{O}_{5}: \mathrm{K}_{2} \mathrm{O}$ ha $^{-1}$

$\mathrm{T}_{14}$ : Absolute control

BDLME - Bio-Digester Liquid Manure

Equivalent 
While, at Kathalagere, application of FYM $7.5 \mathrm{t} \mathrm{ha}{ }^{-1}+$ cattle urine (equivalent to $75 \mathrm{~kg}$ $\mathrm{N} \mathrm{ha}{ }^{-1}$ ) had significantly higher available nitrogen, phosphorus and potassium $(293,27$ and $216 \mathrm{~kg} \mathrm{ha}^{-1}$, respectively) as compared to recommended practice (FYM $10 \mathrm{t}+100: 50: 50$ NPK kg ha $\left.{ }^{-1}\right)(272,21$ and 182) after harvest of rice. Hanumathappa et al., (2012) in coastal zone of Bramhavar, found that application of FYM $10 \mathrm{t} \mathrm{ha}^{-1}+$ cattle urine equivalent to $75 \mathrm{~kg} \mathrm{ha}^{-1}$ increased the soil organic carbon, available phosphorus and potassium $\left(1.19 \%, 296 \mathrm{~kg} \mathrm{ha}^{-1}\right.$ and $195 \mathrm{~kg} \mathrm{ha}^{-}$ ${ }^{1}$, respectively) as compared to initial status (0.55, 180 and 135). Surekha et al., (2011) found that, there was an increase in soil organic carbon, available N, P and $\mathrm{K}$ by 28,7 , 21 , and 21 per cent with organics as compared to inorganics at the end of fourth year.

\section{Soil microbial population}

Significantly higher population of bacteria (24.2 cfu X $10^{5} \mathrm{~g}^{-1}$ of soil), fungi (16.4 cfu X $10^{3} \mathrm{~g}^{-1} \mathrm{~s}$ of soil) and actinomycetes (11.2 cfu $\mathrm{X} 10^{4} \mathrm{~g}^{-1}$ of soil) after harvest of aerobic rice was found with FYM $12.5 \mathrm{t}+$ BDLME to 150 $\mathrm{kg} \mathrm{N} \mathrm{ha}{ }^{-1}$ as compared to the recommended practice but was on par with FYM $12.5 \mathrm{t}+$ BDLME to $125 \mathrm{~kg} \mathrm{~N}^{-1}$ (Table 3). In the same context, higher population of bacteria $\left(25.8 \mathrm{cfu} \times 10^{5} \mathrm{~g}^{-1}\right.$ soil), fungi (17.4 cfu X $10^{3}$ $\mathrm{g}^{-1}$ soil) and actinomycetes (12.2 cfu X $10^{4} \mathrm{~g}^{-1}$ soil) after harvest of field bean were found with the application of FYM $10 \mathrm{t}+$ BDLME to $35 \mathrm{~kg} \mathrm{~N} \mathrm{ha}{ }^{-1}$ as compared to that of recommended practice but was on par with FYM $10 \mathrm{t}+$ BDLME to $30 \mathrm{~kg} \mathrm{~N}^{-1}$ but, was on par with FYM $10 \mathrm{t}+$ BDLME to $30 \mathrm{~kg} \mathrm{~N}$ ha $^{-1}$ (Table 4).

The possible reason for relatively higher rate of multiplication of bacteria in FYM treated plot is that as FYM acted as organic substrate for stimulation of bacterial growth. Moreover, in the present investigation, the population of bacteria, fungi and actinomycetes were higher under field bean than under aerobic rice crop. This might be due to the fact that as field bean is a legume component which is known to release a part of unused $\mathrm{NO}_{3}$ fixed through symbiotic nitrogen fixation into the soil and also a lot of low molecular weight organic compounds are released to the soil as exudates. These serve as a substrate to soil microbes and their population builds-up in the soil (Masood Ali et al., 2002). These results are in conformity with findings of Badole and More (2001) who reported that application of FYM $25 \mathrm{t} \mathrm{ha}^{-1}$ recorded higher population of Rhizobium, Azatobactor, fungi, actinomycetes, PSB and bacteria $\left(10.5 \times 10^{3}\right.$, $0.38 \times 10^{3}, 6.7 \times 10^{3}, 14.9 \times 10^{4}, 29.1 \times 10^{4}$ and $62.5 \times 10^{4}$ cells $\mathrm{g}^{-1}$ soil, respectively) as compared to control treatment.

Field trials at Kathalagere revealed that population of total bacteria $\left(63.6 \times 10^{6} \mathrm{cfu} \mathrm{g}^{-1}\right.$ soil), fungi $\left(34 \times \quad \times 0^{4} \quad \mathrm{cfu}^{-1} \quad\right.$ soil $)$, actinomycetes $\left(53.7 \times \quad 10^{4} \quad \mathrm{cfu}^{-1} \quad \mathrm{~g}^{-1}\right.$ soil $)$, nitrogen-fixers $\left(59.2 \times 10^{5} \mathrm{cfu} \mathrm{g}^{-1}\right.$ soil) and Psolublizers $\left(51.9 \times 10^{5} \mathrm{cfu} \mathrm{g}^{-1}\right.$ soil) were maximum with FYM $12.5 \mathrm{t} \mathrm{ha}^{-1}+$ cattle urine (equivalent to $125 \mathrm{~kg} \mathrm{~N}^{-1}$ ) and minimum of the same was found with recommended practice (FYM $10 \mathrm{t}+$ 100:50:50 NPK kg ha ${ }^{-1}$ ). Similarly, at the end of third year of organic farming in Naganahally, phenomenal increment in the population of Rhizobium, Azotobactor, Azospirillum and PSB's in irrigated soils was observed. Mean increase in Rhizobium from 0.4 to $3.4 \mathrm{cfu} \times 10^{6}$ per g soil. Similarly, Azotobactor, Azospirillum and PSB's increment was 0.8 to $2.2,2.1$ to 3.1 and 1.7 to $3.9 \mathrm{cfu} \times 10^{6}$ per $\mathrm{g}$ soil, respectively (Reddy et al., 2010 and 2011). Further, in 30 years farming system trial at Rodale institute, USA, the soil health was highly improved in terms of soil aggregation, porosity, water holding capacity, nutrient balance and soil microbial biomass (Anon., 2011). 


\section{Acknowledgement}

I am extremely thankful to Zonal Agricultural Research Station, Mandya, Karnataka for providing basic facilities during the course of investigation.

\section{References}

Anonymous. 2011. The Farming Systems Trial. The Rodale Institute, Kutztown, Pensyluvania, USA.

Badole, S.B. and More, S.D. 2001,.Residual effect of nutrient management on yield of groundnut. J. Maharashtra Agric. Univ., 26(1): 109-110.

Dikshit, P.R. and Khatik, S.K. 2002. Influence of organic manures on production, quality and economic feasibility of soybean in typic haplustert of Jabalpur. Legume Res., 25(1): 53-56.

Gajanana, G.N., Ganapathi and shankar, M.A. 2005. Relevance of organic matter for sustainable crop production in dry land - A success story for 25 years. All India coordinated Research Project for dry land agriculture, Univ. Agric. Sci., Bangalore. Pp. 52-57.

Gopalakrishnan, B. and Palaniappan, S.P. 1992. Influence of mussorie rock phosphate on available nutrients in a soybean - sunflower cropping system. $J$. Indian Soc. Soil Sci., 40: 474-476.

Hanumanthappa, H., Sudhir Kamat and Anand, M.R. 2012. Standardization of organic nutrient management under SRI and conventional methods of paddy cultivation. Ann. Prog. Report, Research Institute on Organic Farming, Univ. Agric. Sci., Bangalore. Pp. 56-64.

Jackson, M.L. 1967. Soil Chemical Analysis, Prentice Hall of India Pvt. Ltd., New Delhi. p. 498.
Masood Ali, Ganeshmurhy A.N. and Srinivasara, O. 2002. Role of pulses in soil health and sustainable crop production. Indian J. Pulse Res., 15(2): $107-117$.

Morey, D.K., and Bagde, M.G. 1982. Effect of kharif legumes on yield, nitrogen economy of succeeding wheat and economics of cropping systems. $J$. Maharashtra Agric. Univ., 7(1): 26-29.

Naveed, I.K., Asmat, U.M., Farah, U. and Irfan, B.M. 2010. Effect of tillage and farmyard manure on physical properties of soil. Int. J. Plant Sci., 1(4): 75-82.

Rajnish, S. and Subhash, C. 2011. Performance of basmati rice (Oryza sativa L.) based cropping systems under different modes of nutrient management. Indian J. Agric. Sci., 81(4): 336-339.

Rajshree, M.W., Maya, M.R., Swati, V.W. and Bharti, S.B. 2005. Residual effect of long-term application of FYM on soil properties of Vertisols, yield, protein and oil content of soybean. J. Soils and Crops, 15(1): 155-159.

Reddy, V.C., Jayarama Reddy, M., Shivanandanam, V., Govindaraju, C., Yogananda, S.B., Vijayalakshman, Pradeep, S., Girijesh, G.K. and Hanumanthappa, H. 2011, Developing organic package of practices for production of paddy, ragi, maize, ground nut, red gram, field bean and soybean. Ann. Prog. Report, Research Institute on Organic Farming, Univ. Agric. Sci., Bangalore. Pp. 13-99. 
Reddy, V.C., Shivanandanam, V., Jayarama Reddy, M., Sannathimmappa, M., Bhairappanavar, S.T., Girijesh, G.K., Narayanprasad, Mohan, G.S., Vasanthkumar, H.L., Govindaraju, C., Jagadeesh, B.R., Basavaraj Naik, T.C. and Hanumanthappa, H. 2010. Developing organic package of practices for production of paddy, ragi, maize, ground nut, red gram, field bean and soybean. Ann. Prog. Report, Research Institute on Organic Farming,
Univ. Agric. Sci., Bangalore. Pp. 26127.

Subbaiah, B.Y. and Asija, G.L. 1959. A rapid procedure for the estimation of available nitrogen in soils. Curr. Sci., 25: $259-260$.

Surekha, K., Jhansilakshmi, V., Somasekhar, N., Latha, P.C., Kumar, R.M., Shobha Rani, N., Rao, K.O. and Viraktamath, B.C. 2011. Status of organic farming and research experiences in rice. $J$. Rice Res., 3(1): 23-35.

\section{How to cite this article:}

Siddaram, V.C. Reddy and Krishna Murthy, N. 2017. Effect of Farmyard Manure and Biodigester Liquid Manure on Soil Health under Aerobic Rice - Field Bean Cropping Sequence. Int.J.Curr.Microbiol.App.Sci. 6(5): 684-693. doi: https://doi.org/10.20546/ijcmas.2017.605.078 